\title{
Safety and efficacy of faecal microbiota transplantation for active peripheral psoriatic arthritis: an exploratory randomised placebo- controlled trial
}

\author{
Maja Skov Kragsnaes, ${ }^{1,2}$ Jens Kjeldsen $\odot,{ }^{3}$ Hans Christian Horn $\odot,{ }^{1}$
} Heidi Lausten Munk $\odot,{ }^{1}$ Jens Kristian Pedersen $\odot{ }^{4}$ Søren Andreas Just, ${ }^{4}$ Palle Ahlquist, ${ }^{5}$ Finn Moeller Pedersen, ${ }^{3}$ Maarten de Wit $\odot{ }^{6}{ }^{6}$ Sören Möller $\oplus^{2,7}$ Vibeke Andersen $\odot^{8,9}$ Karsten Kristiansen $\odot \bullet^{10,11}$ Dorte Kinggaard Holm $\odot, 12$ Hanne Marie Holt, ${ }^{13}$ Robin Christensen $\odot{ }^{2,14}$ Torkell Ellingsen $\oplus^{1,2}$

\section{Handling editor Josef $S$ Smolen \\ - Additional supplemental material is published online only. To view, please visit the journal online (http://dx.doi. org/10.1136/annrheumdis- 2020-219511).}

For numbered affiliations see end of article.

\section{Correspondence to} Professor Torkell Ellingsen, Rheumatology Research Unit, Department of Rheumatology, Odense University Hospital,

Odense, Denmark:

torkell.ellingsen@rsyd.dk

Received 13 November 2020 Revised 12 April 2021 Accepted 14 April 2021 Published Online First 29 April 2021
Check for updates

(C) Author(s) (or their employer(s)) 2021. No commercial re-use. See rights and permissions. Published by BMJ.

To cite: Kragsnaes MS,

Kjeldsen J, Horn HC,

et al. Ann Rheum Dis

2021;80:1158-1167.

\section{ABSTRACT}

Objectives Although causality remains to be established, targeting dysbiosis of the intestinal microbiota by faecal microbiota transplantation (FMT) has been proposed as a novel treatment for inflammatory diseases. In this exploratory, proof-ofconcept study, we evaluated the safety and efficacy of FMT in psoriatic arthritis (PsA).

Methods In this double-blind, parallel-group, placebocontrolled, superiority trial, we randomly allocated (1:1) adults with active peripheral PsA ( $\geq 3$ swollen joints) despite ongoing treatment with methotrexate to one gastroscopic-guided FMT or sham transplantation into the duodenum. Safety was monitored throughout the trial. The primary efficacy endpoint was the proportion of participants experiencing treatment failure (ie, needing treatment intensification) through 26 weeks. Key secondary endpoints were change in Health Assessment Questionnaire Disability Index (HAQ-DI) and American College of Rheumatology (ACR20) response at week 26.

Results of 97 screened, 31 (32\%) underwent randomisation (15 allocated to FMT) and 30 (97\%) completed the 26-week clinical evaluation. No serious adverse events were observed. Treatment failure occurred more frequently in the FMT group than in the sham group (9 $(60 \%)$ vs $3(19 \%)$; risk ratio, 3.20; $95 \% \mathrm{Cl}$ 1.06 to $9.62 ; p=0.018)$. Improvement in HAQ-DI differed between groups $(0.07$ vs 0.30$)$ by 0.23 points $(95 \% \mathrm{Cl}$ 0.02 to $0.44 ; p=0.031$ ) in favour of sham. There was no difference in the proportion of ACR20 responders between groups (7 of $15(47 \%)$ vs 8 of $16(50 \%)$ ).

Conclusions In this first preliminary, interventional randomised controlled trial of FMT in immune-mediated arthritis, we did not observe any serious adverse events. Overall, FMT appeared to be inferior to sham in treating active peripheral PsA.

Trial registration number NCT03058900.

\section{INTRODUCTION}

For a century, the link between enteric infections and reactive arthritis ${ }^{1}$ has motivated investigation into the proposed gut-joint axis implicating intestinal micro-organisms in the aetiology of

\section{Key messages}

What is already known about this subject?

- Psoriatic arthritis (PsA) is a systemic immunemediated disease associated with subclinical gut inflammation and dysbiosis of the intestinal microbiota.

- Faecal microbiota transplantation (FMT) has demonstrated local therapeutic immunemodulating abilities in patients with chronic inflammatory bowel disease.

What does this study add?

- In this first preliminary, randomised controlled trial of FMT in immune-mediated arthritis, transfer of donor microbiota was safe, but appeared inferior to sham in reducing disease activity in patients with active peripheral PsA concomitantly treated with methotrexate.

How might this impact on clinical practice or future developments?

- Whether microbial dysbiosis or specific bacteria are common or decisive mediators of disease activity in PsA and whether this proposed relation can be modified without exacerbating the disease will be crucial to clarify to determine the future role of microbiota-targeted interventions in the management of PsA.

immune-mediated arthritic disease. ${ }^{2}$ Recently, this theory has gained renewed interest due to accumulating evidence of disease-related imbalance (dysbiosis) in the composition and function of the intestinal microbiota in chronic disorders. ${ }^{3-5}$ Among these, psoriatic arthritis (PsA) ${ }^{6}$ has been associated with decreased intestinal bacterial diversity, displaying both disease-specific patterns ${ }^{7}$ and microbial abnormalities similar to those seen in other subtypes of spondyloarthritis, rheumatoid arthritis and inflammatory bowel disease (IBD). ${ }^{8}$ These findings have encouraged research into the host-microbiota interplay in the dysregulated 
immunological cascade underlying immune-mediated arthritis and the prospects of microbiota-targeted therapies. ${ }^{9}$

Faecal microbiota transplantation (FMT) is currently considered the most efficient method to restore a healthy diversity of the gastrointestinal microbiota. ${ }^{10}{ }^{11}$ Indeed, the transfer of faeces containing minimally manipulated communities of micro-organisms from a donor to a recipient has revolutionised the treatment of Clostridioides difficile infection. ${ }^{12}$ FMT may also induce beneficial responses in patients with IBD, thereby demonstrating local therapeutic immune-modulating abilities. ${ }^{13}$ However, whether manipulation of the intestinal microbiota can treat extraintestinal, immune-mediated disorders remains to be established. ${ }^{14}$ This is the first exploratory, randomised trial to assess the safety and efficacy of FMT in patients with active, peripheral PsA.

\section{METHODS}

\section{Trial design}

This is a proof-of-concept, 26-week, 1:1 randomised, parallelgroup, double-blind, placebo-controlled, single-centre superiority trial. In 2015, the Regional Committees on Health Research Ethics for Southern Denmark (DK-S-20150080) and the Danish Data Protection Agency (15/41684) approved the trial protocol (see online supplemental appendices S1 and S1A). Although not required by the Danish Health and Medicines Authority, we fulfilled the requirements of documentation, monitoring and reporting according to the principles of Good Clinical Practice. We registered the trial with ClinicalTrials.gov. Our trial protocol paper was published in $2018 .{ }^{15}$ The study was conducted at one Danish tertiary referral hospital with nationwide inclusion. All participants gave written informed consent. The trial was temporarily suspended from March to May 2020 due to the COVID-19 pandemic (see online supplemental appendix S4). A statistical analysis plan (see online supplemental appendix S2) was developed with subsequent closure (2 April 2020) before unmasking and analysis (10 June 2020).

\section{Participants}

We included adults who were between 18 and 75 years of age, fulfilled the Classification for Psoriatic Arthritis criteria, ${ }^{16}$ and had active peripheral disease, defined as three or more swollen joints, despite ongoing treatment with methotrexate at the maximal tolerable dose ( $\geq 15 \mathrm{mg} / \mathrm{week}$ ) for at least 3 months prior to study inclusion. A washout period of 12 weeks (26 weeks for biologic agents) was required in patients previously treated with intra-articular or systemic glucocorticoids, and non-methotrexate conventional synthetic and biologic diseasemodifying antirheumatic drugs. Key exclusion criteria were immune-mediated arthritis other than PsA, IBD, cancer, severe chronic infection, and history of food allergy, severe food intolerance or coeliac disease. ${ }^{15}$

\section{Donor selection and stool preparation}

The transplants were obtained from four healthy stool donors recruited from a non-profit, public stool bank located at the local blood and tissue transplant service. ${ }^{17}$ Every step of the donation process and the laboratory handling were in agreement with the requirements of the European Union's regulative directives on human cells and tissues (2004/23/EC). Donors did not receive any compensation and had to pass an extensive screening programme (see online supplemental table S3) before and after the 30-day donation cycle. ${ }^{15}$ Stool donations were transported to the stool bank facility within 1 hour after defaecation in an airtight container placed in a cooling bag. The donation was processed at normal room temperature within 2 hours of delivery under aerobic conditions, including 10-15 s of blending, before storage at $-80^{\circ} \mathrm{C}$ (median storage time: 20 months; range: $2.5-30) .^{18}$

\section{Interventions}

The transplant consisted of a single stool donation $(50 \mathrm{~g})$ mixed with saline $(0.9 \%)$ and glycerol $(10 \%)$ to a total volume of 250 $\mathrm{mL}$. Before transplantation, we thawed the material to $36^{\circ} \mathrm{C}$. The sham transplant consisted of $250 \mathrm{~mL}$ saline $(0.9 \%)$ mixed with three drops of food colouring (E150c). We performed the allocated treatment within 14 days of the baseline visit. Treatment preparation included a 6-hour fast and one dose of oral proton-pump inhibitor. The transplant suspension was transferred into the third part of the duodenum via a closed system of tubes under gastroscopic guidance.

\section{Outcomes}

Safety was monitored by open assessment of adverse events (AEs) and evaluated before unmasking. The National Cancer Institute Common Terminology Criteria for Adverse Events version 5.0 was used to grade the severity. ${ }^{19}$ We evaluated causality for expected AEs of grade 2 and above and for all unexpected AEs regardless of severity. The primary efficacy endpoint was a composite outcome on the proportion of participants who experienced treatment failure through 26 weeks, defined as need for at least one of the following: more than one intra-articular glucocorticoid injection, and non-methotrexate conventional synthetic and/or biologic disease-modifying antirheumatic drugs. This endpoint covered disease activity and shared decisionmaking between the patient and the rheumatologist in accordance with the European PsA recommendations. ${ }^{20}$ Three key secondary endpoints were evaluated at week 26: change from baseline in Health Assessment Questionnaire Disability Index (HAQ-DI), ${ }^{21}$ proportion of participants fulfilling the American College of Rheumatology (ACR20) response ${ }^{22}$ and change from baseline in the Spondyloarthritis Research Consortium of Canada (SPARCC) Enthesitis Index. ${ }^{23}$ Additional secondary outcomes addressed all essential domains in the PsA core outcome set ${ }^{24}$ (see table 1).

\section{Randomisation and masking}

We assigned participants to FMT or sham transplantation using permuted blocks with varying sizes of four and six, according to computer-generated random numbers. These lists were generated by the trial statistician and uploaded to a concealed area of a password-protected trial database $\left(\right.$ REDCap $\left.^{25}\right)$ by an independent and otherwise trial agnostic data manager. ${ }^{15}$ The trial coordinator implemented the randomisation. The randomisation record and the signed transfusion journals were stored in the database with restricted access separate from the patient record and other study data so the participants and the treating rheumatologists (ie, care providers and outcome assessors) remained unaware of treatment allocation and treatment.

\section{Sample size and power considerations}

Conceptually guided by the idea that at least twice as many participants in the sham group would be treatment failures, compared with the FMT group if the procedure should potentially be considered clinically relevant, we wanted to randomly assign 80 patients with PsA to two groups (40 patients to each), providing a good statistical power $(90 \%)$ to detect a difference 
Table 1 Comparison of efficacy endpoints at week 26

\begin{tabular}{|c|c|c|c|}
\hline Endpoint & FMT $(n=15)$ & Sham $(n=16)$ & $\begin{array}{l}\text { Difference between groups } \\
(95 \% \mathrm{Cl})\end{array}$ \\
\hline \multicolumn{4}{|l|}{ Primary efficacy endpoint } \\
\hline Treatment failure (primary endpoint), $\mathrm{n}(\%) \dagger \ddagger$ & $9(60)^{*}$ & $3(19)$ & 3.20 (1.06 to 9.62$)$ \\
\hline \multicolumn{4}{|l|}{ Components of failure } \\
\hline Total patients receiving $>1$ IA glucocorticoid injection, $\mathrm{n}(\%) \ddagger$ & $2(13)$ & $1(6)$ & $2.13(0.22$ to 21.17$)$ \\
\hline $\begin{array}{l}\text { Total patients starting non-methotrexate conventional synthetic DMARD(s), n } \\
(\%) \neq\end{array}$ & $0(0)$ & $1(6)$ & 0 (NA) \\
\hline Total patients starting biologic DMARD(s), n (\%)‡ & $8(53)$ & $3(19)$ & 2.84 (0.92 to 8.76$)$ \\
\hline \multicolumn{4}{|l|}{ Key secondary efficacy endpoints } \\
\hline Change from baseline in HAQ-DI score§ף & $-0.07(-0.22 \text { to } 0.09)^{*}$ & $-0.30(-0.44$ to -0.15$)$ & $0.23(0.02$ to 0.44$)$ \\
\hline ACR20 response, $n(\%) \ddagger^{* *}$ & $7(47)$ & $8(50)$ & 0.93 (0.45 to 1.94$)$ \\
\hline Change from baseline in SPARCC Enthesitis Index scoret†ł‡ & $-1.9(-3.5$ to -0.3$)$ & $-4.3(-5.8$ to -2.8$)$ & $2.3(0.1$ to 4.5$)$ \\
\hline \multicolumn{4}{|l|}{ Other secondary efficacy endpoints } \\
\hline Modified PsARC response $\mathrm{n}(\%) \neq \S \S$ & $11(73)$ & $13(81)$ & 0.90 (0.61 to 1.33$)$ \\
\hline Change from baseline in DLQI scoreף, Iा *** & $-1.5(-3.5$ to 0.5$)$ & $0.8(-0.9$ to 2.4$)$ & $-2.2(-4.9$ to 0.4$)$ \\
\hline Change from baseline in PASI score $¥ * * * \dagger \dagger \dagger$ & $0.1(-3.7$ to 3.9$)$ & $0.3(-2.4$ to 2.9$)$ & $-0.2(-4.8$ to 4.5$)$ \\
\hline Change from baseline in the number of digits affected with dactylitis $¥ \ddagger^{* * *}$ & $-1.2(-2.1$ to -0.2$)$ & $-1.5(-2.7$ to -0.4$)$ & $0.3(-1.2$ to 1.8$)$ \\
\hline ACR50 response, $\mathrm{n}(\%) \ddagger^{* *}$ & $3(20)$ & $8(50)$ & 0.40 (0.13 to 1.23$)$ \\
\hline ACR70 response, $n(\%) \ddagger^{* *}$ & $1(7)$ & $6(38)$ & 0.18 (0.02 to 1.31$)$ \\
\hline Change from baseline in patient's global assessment, VAS & $-4.3(-14.8$ to 6.3$)$ & $-25.6(-35.4$ to -15.7$)$ & $21.3(6.9$ to 35.7$)$ \\
\hline Change from baseline in arthritis pain, VAS $¥ \neq \neq \ddagger$ & $-8.8(-19.1$ to 1.6$)$ & $-24.8(-34.6$ to -15.0$)$ & $16.0(1.8$ to 30.3$)$ \\
\hline Change from baseline in fatigue, VASq $\neq \neq \ddagger$ & $-0.0(-11.5$ to 11.5$)$ & $-18.1(-29.0$ to -7.2$)$ & $18.0(2.2$ to 33.9$)$ \\
\hline Change from baseline in tender joint count $\ddagger \ddagger$ & $-5.2(-9.8$ to -0.6$)$ & $-9.9(-14.2$ to -5.5$)$ & $4.72(-1.6$ to 11.0$)$ \\
\hline Change from baseline in C reactive protein§§§ & $0.4(-1.0$ to 1.7$)$ & $-1.0(-2.3$ to 0.2$)$ & $1.4(-0.4$ to 3.2$)$ \\
\hline
\end{tabular}

All analyses were performed according to the intention-to-treat principle.

${ }^{*} \mathrm{P}<0.05$ for comparison with sham. Statistical testing was stopped following ACR20 in accordance with the predefined testing hierarchy.

tTreatment failure was defined as need for at least one of the following: more than one intra-articular glucocorticoid injection, and non-methotrexate conventional synthetic and/or biologic disease-modifying antirheumatic drugs.

$\ddagger$ Comparison was calculated as risk ratios based on crude relative risk.

$\S H A Q-D I$ score ranges from 0 to 3 , with higher scores indicating greater disability.

ףComparison was calculated as least squares means based on repeated measures linear mixed model across time points (baseline and weeks 1,2,3,4,12 and 26).

${ }^{* *}$ ACR20/50/70 response ( $\geq 20 \% / 50 \% / 70 \%$ improvement from baseline in the number of tender and swollen joints and in at least 3 of 5 other specified domains).

THSPARCC Enthesitis Index score ranges from 0 to 16 , with higher scores indicating more severe disease.

¥ Comparison was calculated as least squares means based on repeated measures linear mixed model across time points (baseline and weeks 12 and 26).

$\S \S$ Modified PsARC response (two of the following, one of which has to be a tender (68) and swollen (66) joint count, and no worsening of any measure: tender or swollen joint count improvement of $30 \%$ and/or patient global or physician global improvement of at least 1 point on the 5-point Likert scale).

IIDLQI score ranges from 0 to 30 , with higher scores indicating more severe disease.

***PASI score, DLQI score and dactylitis count only in patients with baseline value $>0$.

†††PASI score ranges from 0 to 72 , with higher scores indicating more severe disease.

$\ddagger \neq \neq V A S$ of $0-100$, with higher scores indicating greater disease activity or pain.

$\S \S \S$ Comparison was calculated as least squares means based on repeated measures linear mixed model across time points (baseline and weeks 4,12 and 26).

ACR, American College of Rheumatology; DLQI, Dermatology Life Quality Index; DMARD, disease-modifying antirheumatic drug; FMT, faecal microbiota transplantation; HAQ-DI, Health Assessment Questionnaire Disability Index; IA, intra-articular; NA, not available; PASI, Psoriasis Area Severity Index; PsARC, Psoriatic Arthritis Response Criteria; SPARCC, Spondyloarthritis Research Consortium of Canada; VAS, Visual Analogue Scale.

between two proportions (35\% vs $70 \%$ ) with a significance level of $5 \%$ (see further details in online supplemental appendices S1 and S2). In April 2019, we decided to stop recruitment by 31 December 2019, thereby adhering to the original planned trial completion date of 1 July 2020 (see online supplemental appendix S3). The main reason for this was that essential funding would stop following this date. Due to a slower than expected recruitment rate, only 31 participants were enrolled.

\section{Patient involvement}

Patients were directly involved in the design, funding, recruitment, conduct, reporting and dissemination of the study (see description at the end of the manuscript).

\section{Statistical analysis}

Analyses were based on the intention-to-treat (ITT) population including all randomised individuals, independent of subsequent adherence to the trial protocol. To assess the effect of FMT on the risk of treatment failure during the 26-week trial, we compared groups using risk ratios with $95 \%$ CIs based on an unadjusted (crude) model. Time-to-treatment failure was analysed based on a Kaplan-Meier plot from baseline to week 26 using Cox regression to estimate the HR with 95\% CI and p value. Primary analyses in the ITT population at week 26 were based on a conservative treatment failure imputation default option for binary outcomes and a mixed-effects repeated measures model for continuous variables. For continuous outcome measures, we modelled between-group differences in outcomes at $0,1,2,3,4,12$ and 26 weeks with mixed-effects models, using time and group as categorical fixed-effect factors, interactions between time and group, random intercepts, and an unstructured covariance matrix; these models are reported as least squares means (and the difference between them) with $95 \%$ CIs. 
All $\mathrm{p}$ values and 95\% CIs were two-sided. We did not apply adjustments for multiplicity, rather we analysed the three key secondary outcomes in a prioritised order with a gatekeeping rule for serial testing. The other secondary outcomes are presented without conducting any formal statistical testing. Safety data are summarised descriptively in the full analysis set. In addition, we conducted a per-protocol analysis (see online supplemental table S1 and online supplemental figure S1) which included participants in whom the assigned transplant was successfully transferred into the third part of the duodenum. In addition, participants were excluded (censored) from the analysis following the day where they were categorised as treatment failures. Results of additional sensitivity analyses (including analyses based on the ITT population with missing data handled with multiple imputation) are presented in online supplemental table S2. We performed analyses using StataSE-64 V.16.1.

\section{RESULTS}

\section{Participants}

We enrolled participants to the trial between 16 May 2017 and 11 December 2019 at one Danish tertiary referral hospital (nationwide recruitment) with 26-week follow-up until 2 June 2020. Of 97 patients screened, 31 (32\%) fulfilled the study requirements and were randomised to FMT or sham transplantation. All received the assigned intervention and 30 (97\%) completed the clinical evaluation at week 26 (figure 1). Demographics and clinical characteristics of the two groups were comparable at baseline (table 2), except for some imbalance in sex and disease duration. Of the 31 participants, $27(87 \%)$ had a personal history of skin psoriasis. A complete list of medication is presented in online supplemental table 4.

\section{Safety}

We observed no serious AEs or deaths in any of the groups. Forty-seven AEs occurred in 14 participants (93\%) receiving FMT, and 53 AEs occurred in 14 participants (88\%) receiving sham. No participants withdrew from the trial due to AEs. In the FMT group, AEs were mainly related to the gastrointestinal tract and included abdominal discomfort, flatulence, nausea and vomiting. One case of diverticulitis was deemed unrelated to FMT because the participant had a history of diverticulitis before trial enrolment and the episode occurred 24 weeks after FMT.

Three AEs were deemed related to the gastroscopic procedure. Of those, facial capillary rupture and uncontrolled defaecation both occurred within an hour of the procedure. The third procedure-related $\mathrm{AE}$ was exacerbation of known asthmatic disease (grade 2) 3 days after the procedure. This participant vomited during the gastroscopy. We did not suspect pulmonary aspiration, and the clinical evaluation (including measures of $\mathrm{C}$ reactive protein) performed by the participant's general practitioner 4 and 7 days after the procedure did not suggest an underlying bacterial aetiology. A complete list of AEs, routine laboratory findings and metabolic changes is presented in table 3.

\section{Efficacy}

During the entire 26 weeks of observation, the rate of the primary outcome (treatment failure) was significantly higher in the FMT than in the sham group (HR, 4.87 (95\% CI 1.31 to 18.18); $\mathrm{p}=0.018$ ) (see figure 2). After 26 weeks, treatment had failed in more FMT-treated participants (9 of 15, 60\%) than sham-treated participants (3 of 16, 19\%) (crude relative risk, 3.20 (95\% CI 1.06 to 9.62); $\mathrm{p}=0.018$ ) (see table 1).

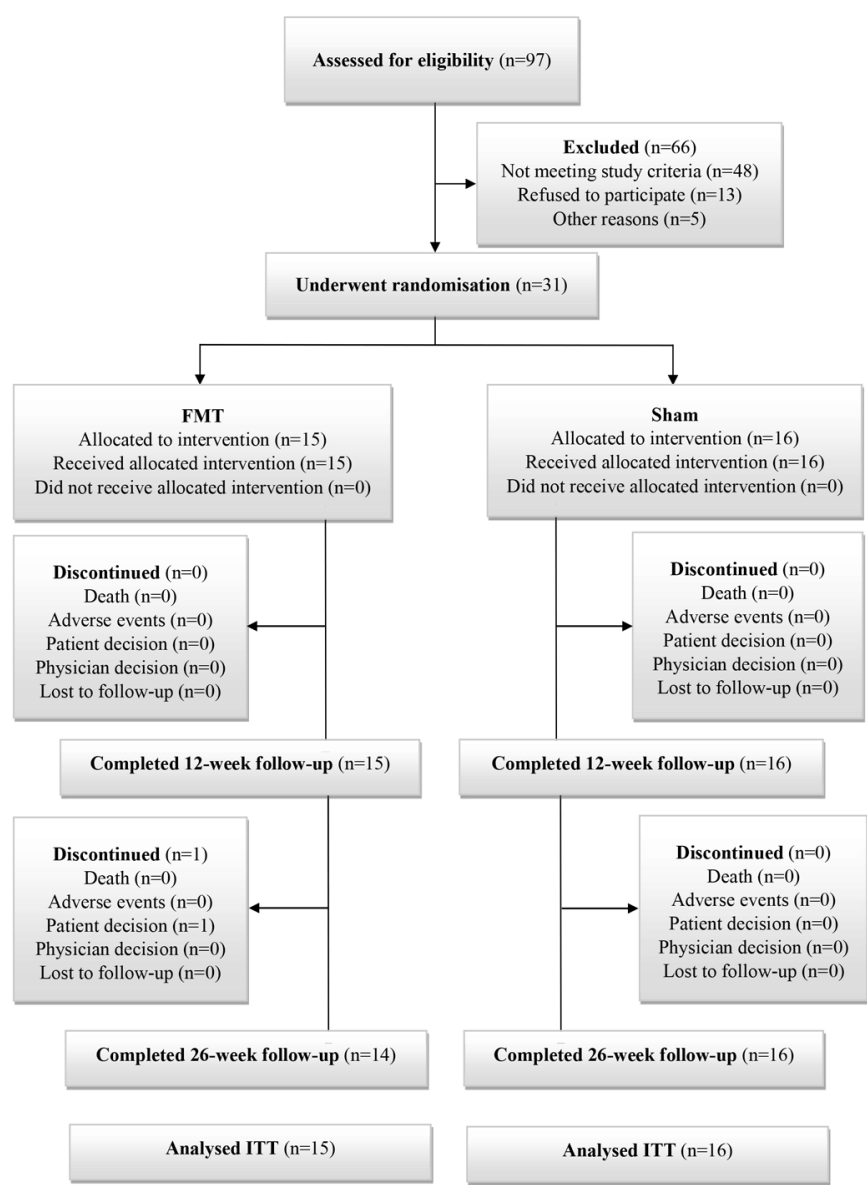

Figure 1 Patient disposition. Reasons for not meeting study criteria $(n=48)$ : not diagnosed with psoriatic arthritis $(n=5)$, not $\geq 3$ swollen joints $(n=11)$, treated with other $\operatorname{csDMARD}(n=2)$ or bDMARD $(n=5)$, methotrexate ( $\geq 15 \mathrm{mg} /$ week) toxicity $(n=6)$, age below or above limit $(n=3)$, systemic inflammatory comorbidity $(n=1)$, living abroad $(n=14)$ and closed inclusion 2 days after initial contact $(n=1)$. bDMARD, biologic disease-modifying antirheumatic drug; CSDMARD, conventional synthetic disease-modifying antirheumatic drug; FMT, faecal microbiota transplantation; ITT, intention-to-treat.

Starting biologic disease-modifying antirheumatic drug was the main reason for being categorised as treatment failure (8 of $15(53 \%)$ vs 3 of $16(19 \%)$ ). In the FMT group, eight patients $(53 \%)$ had initiated biologic therapy at the 12-week visit compared with two (13\%) in the sham group. The median time from trial intervention to starting biologics was 32 days (IQR 23-64) in the FMT group and 99 days (IQR 60-175) in the sham group. The median time from starting biologics to evaluation at week 26 was 155 days (IQR 118-173; total group exposure time, 995 days) in the FMT group and 70 days (IQR 0-126; total group exposure time, 196 days) in the sham group.

The HAQ-DI decreased more (indicating better physical function) in the sham group than in the FMT group (least squares means, $-0.30(95 \% \mathrm{CI}-0.44$ to -0.15$)$ vs -0.07 (95\% CI -0.22 to 0.09 ), difference 0.23 (0.02 to 0.44$) ; p=0.031$ ). The trajectories for HAQ-DI by treatment group from baseline to week 26 are presented in figure 3 . Hierarchical statistical testing failed with regard to the proportion of ACR20 responders when comparing the FMT group with sham (7 of 15 (47\%) vs 8 of 16 (50\%); crude relative risk, 0.93 (0.45 to 1.94$)$ ). 
Psoriatic arthritis

Table 2 Baseline demographics and disease characteristics

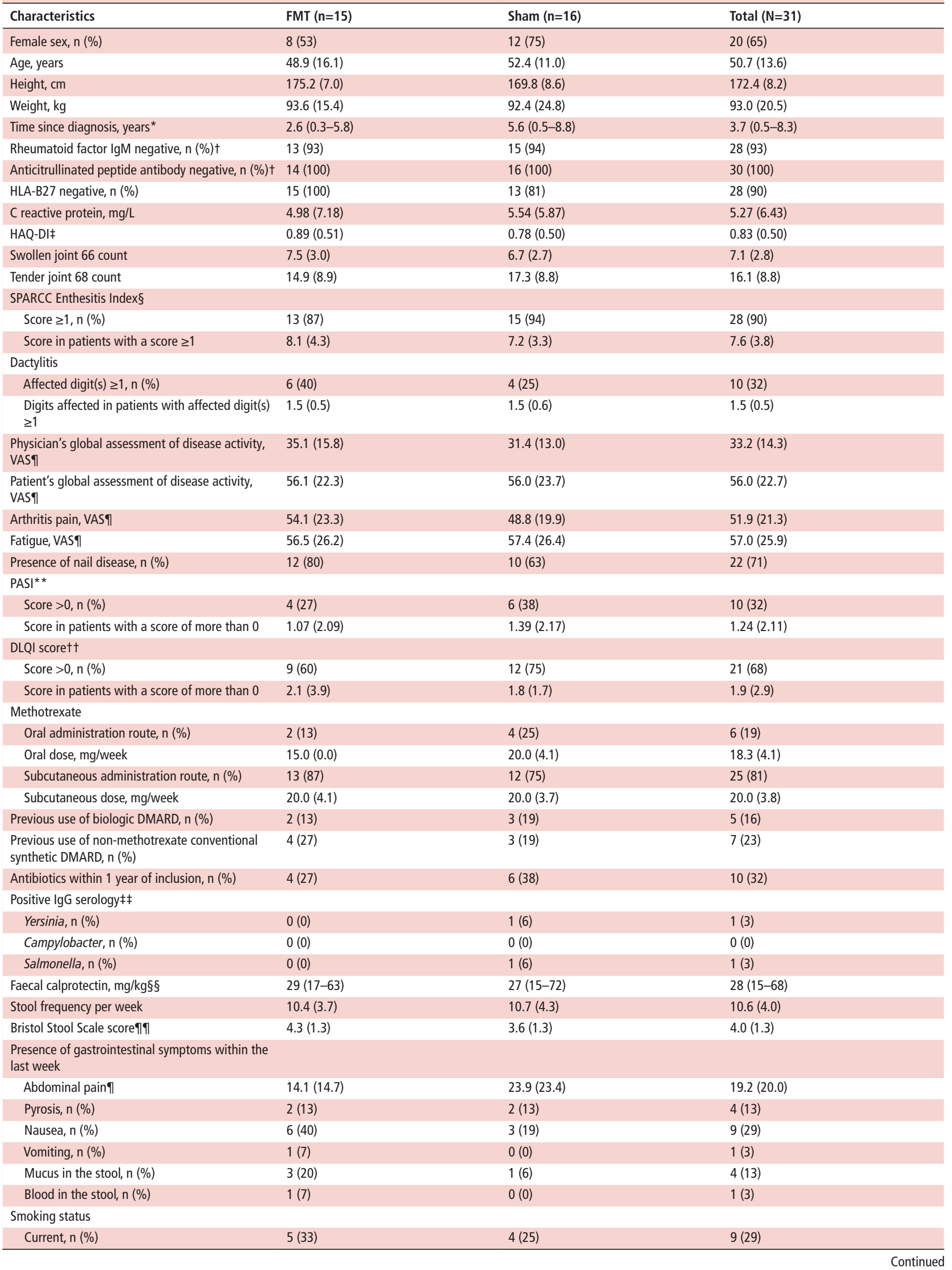


Table 2 Continued

\begin{tabular}{clll}
\hline Characteristics & FMT $(\mathrm{n}=15)$ & Sham $(\mathrm{n}=16)$ & Total $(\mathrm{N}=31)$ \\
\hline Previous, $\mathrm{n}(\%)$ & $4(27)$ & $8(50)$ & $12(39)$ \\
Never, $\mathrm{n}(\%)$ & $6(40)$ & $4(25)$ & $10(32)$ \\
Alcohol consumption, units per week & $0.9(0.9)$ & $0.8(0.6)$ & $0.8(0.7)$ \\
\hline
\end{tabular}

Data are mean (SD) or $\mathrm{n}(\%)$ unless otherwise stated.

*Time since diagnosis of psoriatic arthritis is presented as median and IQR.

†Presence of rheumatoid factor (IgM) and anticitrullinated peptide antibody was not accessed in one patient from the FMT group.

$\neq$ HAQ-DI scores range from 0 to 3, with higher scores indicating greater disability.

§SPARCC Enthesitis Index scores range from 0 to 16 , with higher scores indicating more severe disease.

१This evaluation is based on a VAS of $0-100$, with higher scores indicating greater disease activity or pain.

**PASI scores range from 0 to 72 , with higher scores indicating more severe disease.

††DLQI scores range from 0 to 30, with higher scores indicating more severe disease.

$\ddagger \ddagger$ Serology specification: Salmonella enteritidis and S. typhimurium, Yersinia enterocolitica, and Campylobacter coli and C. jejuni.

$\S \S$ Median (IQR). Lower detectable limit of faecal calprotectin is $15 \mathrm{mg} / \mathrm{g}$.

ๆๆBristol Stool Scale scores range from 1 to 7, with types 1 and 2 indicating constipation, types 3 and 4 normal, and types 5-7 tending towards diarrhoea (loose to watery stool).

DLQI, Dermatology Life Quality Index; DMARD, disease-modifying antirheumatic drug; FMT, faecal microbiota transplantation; HAQ-DI, Health Assessment Questionnaire

Disability Index; HLA-B27, human leukocyte antigen-B27; PASI, Psoriasis Area Severity Index; SPARCC, Spondyloarthritis Research Consortium of Canada; VAS, Visual Analogue Scale.

\section{DISCUSSION}

Modification of the immunogenic, intestinal microbial communities and their metabolites associated with immunemediated diseases has been highlighted as a possible way to either directly or indirectly modulate a dysregulated immune response in the recipient. In this first FMT trial in PsA, we performed one upper, single-donor FMT to evaluate safety and efficacy in patients with active, peripheral PsA treated with steady state dose methotrexate ( $\geq 15 \mathrm{mg} /$ week). Most importantly, one FMT appeared to be safe in this patient population. Contrary to our hypothesis, the rate of treatment failure (ie, patients needing treatment intensification) was significantly higher in the FMT group than in the sham group. Likewise, the HAQ-DI improved more in sham-treated (indicating better physical function) than in FMT-treated patients. ${ }^{26}$ The event curve (figure 2) demonstrates that treatment failure occurred very quickly after the procedure in patients receiving FMT. Because of the comparable disease activity between groups at baseline, our findings suggest that FMT from selected donors can worsen the symptoms of PsA. This contrasts a case report of a patient with PsA where FMT seemed to assert beneficial effects on the arthritic disease. ${ }^{27}$

FMT for other immune-mediated conditions such as IBD has demonstrated local therapeutic immune-modulating abilities, and disease flares following FMT seem to occur in similar rates among control group patients. ${ }^{13}$ Although transient increase in $\mathrm{C}$ reactive protein and self-limiting fever are wellknown side effects associated with an immunological response in patients receiving FMT for $C$. difficile infection and/or $\mathrm{IBD},{ }^{28}$ based on the existing evidence, thoroughly screened stool for FMT is in general considered safe and has not been related to severe immune responses. Hence, our findings add to the growing body of evidence suggesting a gut-joint axis in the pathogenesis of PsA. ${ }^{29} 30$

A strength of our study is that we designed the trial to provide results relevant to clinical practice. This included the use of FMT products from routine treatment of recurrent C. difficile infections, the timing of the intervention, the long follow-up, the allowance of antibiotics and other medication during follow-up (except for non-methotrexate disease-modifying antirheumatic drugs), and the lack of diet restrictions. Due to the randomised design, allocation concealment, masking of patients and treating rheumatologists/outcome assessors, high treatment adherence, low attrition, no missing data on the primary outcome, and only few cases of protocol violations (see online supplemental text S1), we deem the risk of bias to be low. Furthermore, because our decision to stop the trial before reaching 80 patients was made independently of the trial findings, we do not expect that this decision has biased the results. ${ }^{31}$ Nevertheless, the small study population did affect the precision of the trial estimates, making the conclusions less robust.

Limitations include the initiation of additional diseasemodifying antirheumatic drugs in patients experiencing treatment failure, which may likely have exerted positive effects on the secondary outcomes (HAQ-DI, ACR20 and SPARCC Enthesitis Index) evaluated at week 26. Although this may especially be true for the FMT group, where the majority of participants received biologics within the first 12 weeks of the trial, this explanation is not valid in the sham group, where less than one in five received additional treatment. Hence, the significant clinical improvement observed in this group suggests the presence of trial participation effects. ${ }^{32}$ Findings from a qualitative study nested within the trial support this notion. ${ }^{33}$ Nevertheless, based on the double-blind, randomised design and the fairly comparable demographics and disease characteristics of FMT-treated and sham-treated patients at baseline, we have no reason to believe that exposure, intensity and susceptibility of these effects differed between groups. ${ }^{34}$

The compositional nature of the primary endpoint combining both the patient's values, preferences and needs in relation to the outcome domains that were important to him/ her (eg, pain, physical function, fatigue and social participation) with the physician's assessment of disease (eg, musculoskeletal disease activity and systemic inflammation), in addition to the very high ACR50/70 response in the sham group, complicated the interpretation of the trial results. For example, we cannot rule out that parts of patients' perceptions of the disease, and the resulting motivation for receiving additional treatment, could have been affected by elements of the disease not related to active inflammation (which was our hypothesised target of the FMT) such as central sensitisation and structural damage. In addition, while measures of disease activity appeared comparable between groups at baseline, the (random) imbalance in sex and disease duration could hypothetically have had an effect on the between-group differences in the primary and secondary outcomes. 


\begin{tabular}{|c|c|c|}
\hline AE events* & $\begin{array}{l}\text { FMT }(n=15) \\
\text { PYRS }=7.4\end{array}$ & $\begin{array}{l}\text { Sham }(n=16) \\
\text { PYRS }=8.5\end{array}$ \\
\hline Total AEs, $n$ & 57 & 53 \\
\hline Total patients with $\mathrm{AE}, \mathrm{n}(\%)$ & $14(93)$ & $14(88)$ \\
\hline Total SAEs, $\mathrm{n}$ & 0 & 0 \\
\hline $\begin{array}{l}\text { Total patients with SAE, } \\
\mathrm{n}(\%)\end{array}$ & 0 & 0 \\
\hline $\begin{array}{l}\text { Withdrawal due to any } A E_{\text {, }} \\
n(\%)\end{array}$ & 0 & 0 \\
\hline $\begin{array}{l}\text { Withdrawal due to any SAE, } \\
\mathrm{n}(\%)\end{array}$ & 0 & 0 \\
\hline Death, $n(\%)$ & 0 & 0 \\
\hline \multicolumn{3}{|l|}{ AEs of special interest +} \\
\hline $\begin{array}{l}\text { Change from baseline in } \\
\text { abdominal pain, VAS } ₫ \S\end{array}$ & 8.8 (1.9 to 15.7$)$ & $-18.0(-24.5$ to -11.4$)$ \\
\hline Change from baseline in & $-0.2(-0.7$ to 0.3$)$ & $0.0(-0.5$ to 0.5$)$ \\
\hline
\end{tabular}

Bristol Stool Scale score,

types $1-7 \S \rrbracket$

\begin{tabular}{|c|c|c|}
\hline $\begin{array}{l}\text { Change from baseline in } \\
\text { faecal calprotectin, } \mathrm{mg} / \mathrm{kg}^{* *}\end{array}$ & 86 (-60 to 324$)$ & $16(-36$ to 69$)$ \\
\hline $\begin{array}{l}\text { Total episodes of patient- } \\
\text { reported fever, } \mathrm{n}\end{array}$ & 13 & 12 \\
\hline $\begin{array}{l}\text { Patients treated with } \\
\text { antibiotics, } \mathrm{n}(\%)\end{array}$ & $2(13)$ & $5(31)$ \\
\hline Nausea, n (\%) & $9(60)$ & $7(44)$ \\
\hline Reflux, n (\%) & $8(53)$ & $8(50)$ \\
\hline Vomiting, n (\%) & $6(40)$ & $1(6)$ \\
\hline Blood in the stool, $n(\%)$ & $0(0)$ & $1(6)$ \\
\hline Mucus in the stool, $n(\%)$ & $4(27)$ & $0(0)$ \\
\hline $\begin{array}{l}\text { Elevated plasma alanine } \\
\text { aminotransferase (men >70 } \\
\text { U/L; women }>45 \text { U/L), n (\%) }\end{array}$ & $2(13)$ & $5(31)$ \\
\hline \multicolumn{3}{|l|}{ Abnormal white cell count } \\
\hline Low $\left(<3.50 \times 10^{9} / \mathrm{L}\right), \mathrm{n}(\%)$ & $0(0)$ & $0(0)$ \\
\hline High $\left(>8.80 \times 10^{9} / L\right), n(\%)$ & $5(33)$ & $6(38)$ \\
\hline \multicolumn{3}{|l|}{ Abnormal platelet count } \\
\hline $\begin{array}{l}\text { Low }\left(\text { men }<145 \times 10^{9} / \mathrm{L} ;\right. \\
\left.\text { women }<165 \times 10^{9} / \mathrm{L}\right) \\
\mathrm{n}(\%)\end{array}$ & $0(0)$ & $1(6)$ \\
\hline $\begin{array}{l}\text { High }\left(\text { men }>350 \times 10^{9} / L \text {; }\right. \\
\left.\text { women }>400 \times 10^{9} / L\right) \\
n(\%)\end{array}$ & $1(7)$ & $2(13)$ \\
\hline $\begin{array}{l}\text { Low level of haemoglobin } \\
\text { (men }<134 \mathrm{~g} / \mathrm{L} ; \text { women }< \\
118 \mathrm{~g} / \mathrm{L}), \mathrm{n}(\%)\end{array}$ & $3(20)$ & $1(6)$ \\
\hline Change in weight, kgt† & 0.9 (-0.6 to 2.3$)$ & $-0.1(-1.5$ to 1.2$)$ \\
\hline $\begin{array}{l}\text { Change in haemoglobin A1c } \\
\text { level, } \mathrm{mmol} / \mathrm{molt \dagger}\end{array}$ & $1.43(0.35$ to 2.52$)$ & $0.31(-0.70$ to 1.33$)$ \\
\hline $\begin{array}{l}\text { Change in plasma cholesterol } \\
\text { level, mmol/Lt† }\end{array}$ & $0.23(-0.03$ to 0.49$)$ & $-0.08(-0.31$ to 0.16$)$ \\
\hline $\begin{array}{l}\text { Change in plasma low- } \\
\text { density lipoprotein } \\
\text { cholesterol level, mmol/L†† }\end{array}$ & $0.13(-0.08$ to 0.34$)$ & $-0.14(-0.33$ to 0.05$)$ \\
\hline $\begin{array}{l}\text { Change in plasma high- } \\
\text { density lipoprotein } \\
\text { cholesterol level, mmol/L†t }\end{array}$ & $0.05(-0.05$ to 0.15$)$ & $-0.01(-0.10$ to 0.08$)$ \\
\hline $\begin{array}{l}\text { Change in plasma triglyceride } \\
\text { level, mmol/L†† }\end{array}$ & $-0.01(-0.37$ to 0.34$)$ & $0.15(-0.17$ to 0.47$)$ \\
\hline \multicolumn{3}{|l|}{ Other reported $\mathrm{AEs}, \mathrm{n}(\%) \dagger$} \\
\hline Pneumonia & $1(7)$ & $1(6)$ \\
\hline Gastroenteritis & $0(0)$ & $1(6)$ \\
\hline Sinusitis & $0(0)$ & $1(6)$ \\
\hline
\end{tabular}

\begin{tabular}{|c|c|c|}
\hline AE events* & $\begin{array}{l}\text { FMT }(n=15) \\
\text { PYRS }=7.4\end{array}$ & $\begin{array}{l}\text { Sham }(n=16) \\
\text { PYRS }=8.5\end{array}$ \\
\hline Wound infection & $0(0)$ & $1(6)$ \\
\hline Cystitis & $1(7)$ & $1(6)$ \\
\hline Conjunctivitis & $0(0)$ & $1(6)$ \\
\hline Diverticulitis & $1(7)$ & $0(0)$ \\
\hline Influenza symptoms & $0(0)$ & $1(6)$ \\
\hline $\begin{array}{l}\text { Bronchial asthma } \\
\text { exacerbation }\end{array}$ & $1(7)$ & $0(0)$ \\
\hline $\begin{array}{l}\text { Benign paroxysmal positional } \\
\text { vertigo }\end{array}$ & $0(0)$ & $1(6)$ \\
\hline Flatulence & $1(7)$ & $0(0)$ \\
\hline Uncontrolled defaecation & $1(7)$ & $0(0)$ \\
\hline Facial capillary rupture & $0(0)$ & $1(6)$ \\
\hline
\end{tabular}

Full analysis set: all patients who were randomly assigned to a study group and had exposure to the intervention (FMT or sham) independent of group.

*Any AEs: data are number of events or number of patients (\%). Treatment with antibiotics was not counted as a separate AE.

tAEs of special interest and other reported AEs: dichotomous data are number of patients with at least one episode (\%) unless otherwise specified.

\#VAS of $0-100$, with higher scores indicating greater disease activity or pain. $\S$ Abdominal pain and Bristol Stool Scale score are reported as the main effect of group based on a repeated measures linear mixed model across time points (baseline, week 1, week 2, week 3, week 4, week 12 and week 26). १Bristol Stool Scale score ranges from 1 to 7, with types 1 and 2 indicating constipation, types 3 and 4 normal, and types 5-7 tending towards diarrhoea (loose to watery stool).

${ }^{* *}$ Faecal calprotectin is reported as the main effect of group based on a repeated measures linear mixed model (bootstrap SE) across time points (baseline, week 4 , week 12 and week 26).

t†The metabolic markers (weight, haemoglobin A1c, cholesterol and triglyceride) are evaluated as change from baseline to week 26 . Positive values signify an increase, whereas negative values signify a decrease.

$A E$, adverse event; $F M T$, faecal microbiota transplantation; PYRS, person years at risk; SAE, serious adverse event; VAS, Visual Analogue Scale.

Furthermore, the participants of our trial constituted primarily adults with active, polyarticular PsA, which is a relatively rare condition in clinical practice. ${ }^{20}$ Consequently, although the spectrum of patients with PsA that are enrolled in pharmacological trials is skewed towards this study population, the ability to generalise our findings to the majority of patients with PsA is limited. Moreover, because only 10 participants (32\%) had active skin psoriasis at the time of inclusion, this trial was not suited for assessment of the potential of microbiota modulation in cutaneous inflammation. Finally, our study was neither large enough nor long enough to evaluate uncommon serious AEs and long-term risks. ${ }^{35}$

Previous findings in patients with ulcerative colitis indicate that one FMT regimen performed within 1 week is insufficient to maintain long-lasting (12 months) local anti-inflammatory response in the majority of patients with a beneficial response after 8 weeks. ${ }^{36}$ Hence, we could have missed early clinical significant changes in the secondary outcomes that abated before the 26-week evaluation. In addition, pooled donor batches and high-intensity induction of FMT followed by frequent administration of donor transplant seem to enhance the chances for clinical remission in ulcerative colitis. ${ }^{36-38}$ Perhaps as importantly, previous successes of FMT in IBD appear to have been driven by 'super-donors' characterised by the presence or absence of specific bacteria species. ${ }^{36}{ }^{39}$ To further complicate this picture, matching of donor and recipient could be another important factor to consider. 


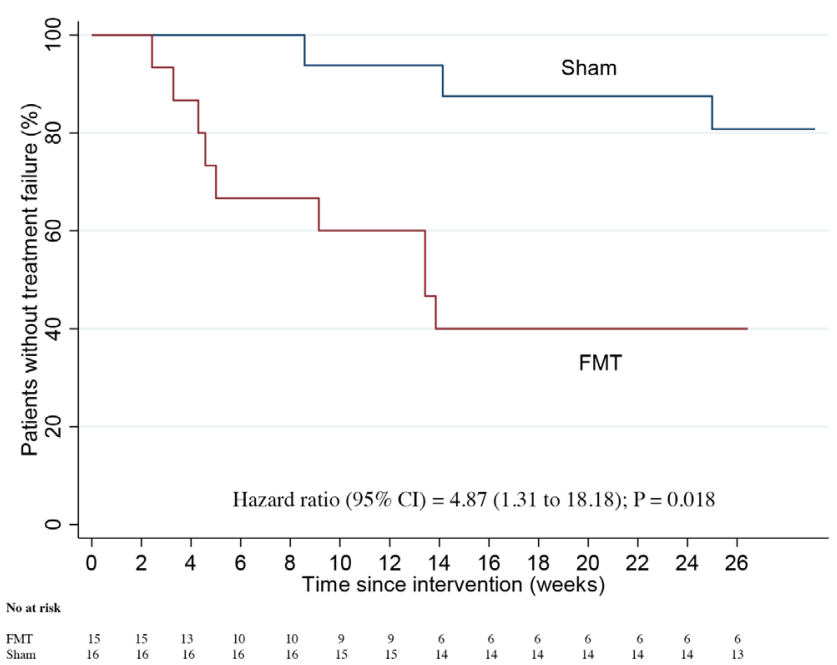

Figure 2 Event curves of treatment failure by intervention group from baseline to week 26. Time-to-treatment failure was analysed using survival analysis as Kaplan-Meier curves from baseline to week 26. Cox regression was used to provide an estimate of the $\mathrm{HR}$ with $95 \% \mathrm{Cl}$ and $p$ value. The number of participants remaining at risk is displayed below the horizontal axis. Treatment failure was defined as need for at least one of the following: more than one intra-articular glucocorticoid injection, and non-methotrexate conventional synthetic and/or biologic disease-modifying antirheumatic drugs. FMT, faecal microbiota transplantation.

In conclusion, further investigation is needed to explore whether extrinsic factors related to the FMT method, such as single versus multiple donor batches, fresh versus frozen products, aerobic versus anaerobic environment, type of stool preparation protocol, storage time, freeze-thaw cycles, pretreatment preparation such as bowel lavage and antibiotics, delivery form, and overall treatment strategy (dose and frequency), may influence the outcome of FMT in PsA. ${ }^{40}$ Future FMT trials could pursue an approach that is similar to the ones used in IBD studies. ${ }^{41}$ Moreover, evaluation of dysbiosis in patients (or donors) prior to trial entry could hypothetically

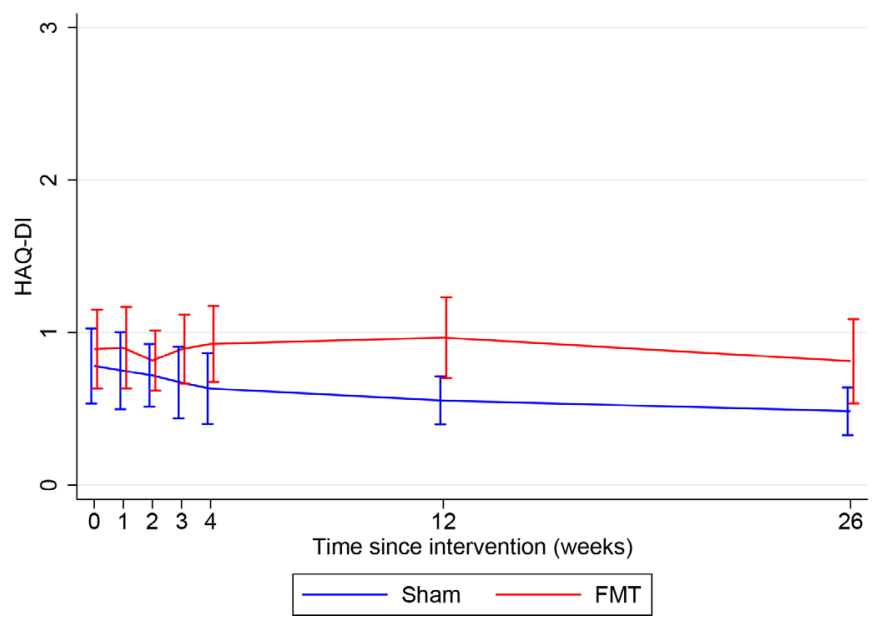

Figure 3 HAQ-DI scores by treatment group from baseline to week 26. Least squares means calculated from the repeated measures linear mixed model: intention-to-treat population. Bars indicate $95 \% \mathrm{Cl}$. HAQ-DI scores range from 0 to 3 , with higher scores indicating greater disability. FMT, faecal microbiota transplantation; HAQ-DI, Health Assessment Questionnaire Disability Index. enhance FMT efficiency. Other mechanisms that should be thoroughly investigated in future studies include the degree and durability of donor microbiota engraftment, changes in patients' microbiota following FMT and comparator intervention, and characterisation of 'good' and 'bad' donations. Indeed, the lack of comprehensive microbiota analyses is a limitation of the current study.

In this preliminary randomised controlled trial with focus on safety, we did not observe any serious AEs. Although no firm conclusions can be drawn from this small trial and despite the similar proportions of ACR20 responders between groups at 26 weeks, our findings indicate that FMT may lead to worsening of PsA, suggesting a role of the intestinal microbiota in downstream immune effects of this disease. Larger, randomised trials of FMT where a sufficient amount of participants will be included combined with exploration of immunological effects and indepth analyses of the composition and functional potential of the microbiota in donor and recipients should be undertaken to further investigate the safety and potential benefits of therapeutic targeting of the gut-joint axis in immune-mediated arthritis.

\section{Patient and public involvement}

Patients and the public played an important part in all elements of the research process beyond the conception of the trial. In the design phase, we asked patients who attended the outpatient clinic to provide input on ethical challenges and trial logistics, especially regarding donor selection and method of FMT administration. Patients also gave feedback to the wording and design of patient information material and recruitment flyers. During the undertaking of the trial, we asked patient organisation and the public to assist the refinement of our recruitment strategy and to help with publicity and funding. Following the last trial visit and before unmasking, we interviewed 10 participants about their trial experiences, which provided insight into their motivation for participation, impact on everyday life, FMT acceptability, and factors related to the trial that may have promoted trial participation effects (the results of this qualitative study will be presented elsewhere). In the dissemination phase of the trial, we invited a patient research partner (MW), who did not participate in the trial, to become coauthor. He made valuable suggestions for improving the reporting of the study and helped us clarify the main findings as seen from a patient's perspective. Finally, we disseminated the results of the trial in a letter to all study participants and invited them to attend an online meeting, where we further explained and discussed the findings of the trial.

\section{Author affiliations}

${ }^{1}$ Rheumatology Research Unit, Department of Rheumatology, Odense University Hospital, Odense, Denmark

${ }^{2}$ Department of Clinical Research, University of Southern Denmark, Odense, Denmark ${ }^{3}$ Department of Medical Gastroenterology, Odense University Hospital, Odense, Denmark

${ }^{4}$ Section of Rheumatology, Department of Medicine, Svendborg Hospital, Svendborg, Denmark

${ }^{5}$ Reumaklinik Fyn, Odense, Denmark

${ }^{6}$ Patient Research Partner, Amsterdam, The Netherlands

${ }^{7}$ OPEN - Open Patient data Explorative Network, Odense University Hospital, Odense, Denmark

${ }^{8}$ IRS-Center Sønderiylland, University Hospital of Southern Denmark, Aabenraa, Denmark

${ }^{9}$ Institute of Molecular Medicine, University of Southern Denmark, Odense, Denmark

${ }^{10}$ Laboratory of Genomics and Molecular Biomedicine, Department of Biology,

University of Copenhagen, Copenhagen, Denmark

${ }^{11}$ Institute of Metagenomics, BGI-Shenzhen, Shenzhen, China

${ }^{12}$ Department of Clinical Immunology, Odense University Hospital, Odense, Denmark 
${ }^{13}$ Department of Clinical Microbiology, Odense University Hospital, Odense, Denmark ${ }^{14}$ Section for Biostatistics and Evidence-Based Research, the Parker Institute, Bispebjerg and Frederiksberg Hospital, Copenhagen, Denmark

Twitter Søren Andreas Just @JustSoren

Acknowledgements We thank all participants for their contribution. We thank CS Klinkby, trial nurse, for assistance in relation to the conduct of the trial visits. We also thank L Albjerg (biomedical laboratory technologist), AC Nilsson (consultant), KF Rasmussen (consultant) and I Georgsen (consultant) at the Department of Clinical Immunology, Odense University Hospital, Denmark, for assisting in the implementation of the FMT stool bank.

Contributors TE, MSK, RC and JK designed the study. MSK and TE were responsible for funding. MSK, DKH and HMH were responsible for donor recruitment, screening and FMT product manufacture. JK and FMP were responsible for the FMT procedure. MSK, TE, HCH, HLM, JKP, PA and SAJ were responsible for patient recruitment. TE, HCH and HLM acquired the clinical data. MSK, SM and RC analysed the clinical data. TE, MSK, RC, JK, HLM, HCH, KK and MW interpreted the results. MSK, TE and RC drafted the report. All authors critically reviewed the report and approved the final version. MSK and TE are guarantors. The corresponding author (TE) attests that all listed authors meet the authorship criteria and that no others meeting the criteria have been omitted.

Funding This study was supported by the Danish Rheumatism Association, the Danish Psoriasis Research Foundation, the University of Southern Denmark Research Fund, the Research Fund of Odense University Hospital, the Danish Regions (Medicinpuljen), the Region of Southern Denmark Research Fund and Novartis Healthcare (unrestricted grant)

Competing interests VA declares personal fees from Merck (MSD) and personal fees from Janssen, outside the submitted work. RC declares a core grant to his institution (Parker Institute, Bispebjerg and Frederiksberg Hospital) from the Oak Foundation (OCAY-18-774-OFIL) and honorariums paid to his institution in relation to the following activities: lecture, research methods (Pfizer, DK; 2017); lecture, GRADE lecture (Celgene, DK; 2017); ad board lecture, CAM (Orkla Health, DK; 2017); project grant: 'GreenWhistle' (Mundipharma, 2019); lecture: diet in RMD (Novartis, DK; 2019); consultancy report, Network MA's (Biogen, DK; 2017); ad board lecture, GRADE (Lilly, DK; 2017); consultancy report, GRADE (Celgene, 2018); and lecture, Network MA's (LEO; 2020).

Patient consent for publication Not required.

Ethics approval The study was approved by the Regional Committees on Health Research Ethics for Southern Denmark (DK-S-20150080).

Provenance and peer review Not commissioned; externally peer reviewed.

Data availability statement Data are available upon reasonable request. Requests on data sharing can be made by contacting the corresponding author. Data will be shared after review and approval by the trial scientific board, and terms of collaboration will be reached together with a signed data access agreement.

Supplemental material This content has been supplied by the author(s). It has not been vetted by BMJ Publishing Group Limited (BMJ) and may not have been peer-reviewed. Any opinions or recommendations discussed are solely those of the author(s) and are not endorsed by BMJ. BMJ disclaims all liability and responsibility arising from any reliance placed on the content. Where the content includes any translated material, BMJ does not warrant the accuracy and reliability of the translations (including but not limited to local regulations, clinical guidelines, terminology, drug names and drug dosages), and is not responsible for any error and/or omissions arising from translation and adaptation or otherwise.

\section{ORCID iDs}

Jens Kjeldsen http://orcid.org/0000-0001-8148-6572

Hans Christian Horn http://orcid.org/0000-0002-0792-0366

Heidi Lausten Munk http://orcid.org/0000-0002-2212-6283

Jens Kristian Pedersen http://orcid.org/0000-0003-2531-9547

Maarten de Wit http://orcid.org/0000-0002-8428-6354

Sören Möller http://orcid.org/0000-0003-0858-4269

Vibeke Andersen http://orcid.org/0000-0002-0127-2863

Karsten Kristiansen http://orcid.org/0000-0002-6024-0917

Dorte Kinggaard Holm http://orcid.org/0000-0002-8156-7177

Robin Christensen http://orcid.org/0000-0002-6600-0631

Torkell Ellingsen http://orcid.org/0000-0003-0426-4962

\section{REFERENCES}

1 Keat A. Reiter's Syndrome and Reactive Arthritis in Perspective. N Engl J Med Overseas Ed 1983;309:1606-15

2 Manasson J, Blank RB, Scher JU. The microbiome in rheumatology: where are we and where should we go? Ann Rheum Dis 2020;79:727-33.

3 Lynch SV, Pedersen 0. The human intestinal microbiome in health and disease. N Engl J Med 2016;375:2369-79.
4 Clemente JC, Manasson J, Scher JU. The role of the gut microbiome in systemic inflammatory disease. BMJ 2018;360:55145.

5 Relman DA. The human microbiome and the future practice of medicine. JAMA 2015:314:1127-8

6 Ritchlin CT, Colbert RA, Gladman DD. Psoriatic arthritis. N Engl J Med 2017:376:957-70.

7 Scher JU, Ubeda C, Artacho A, et al. Decreased bacterial diversity characterizes the altered gut microbiota in patients with psoriatic arthritis, resembling dysbiosis in inflammatory bowel disease. Arthritis Rheumatol 2015;67:128-39.

8 Salem F, Kindt N, Marchesi JR, et al. Gut microbiome in chronic rheumatic and inflammatory bowel diseases: similarities and differences. United European Gastroenterol J 2019;7:1008-32.

9 Mauro D, Ciccia F. Gut dysbiosis in spondyloarthritis: cause or effect? Best Pract Res Clin Rheumatol 2019;33:101493.

10 Ianiro G, Maida M, Burisch J, et al. Efficacy of different faecal microbiota transplantation protocols for Clostridium difficile infection: A systematic review and meta-analysis. United European Gastroenterol J 2018:6:1232-44.

11 Ramai D, Zakhia K, Ofosu A, et al. Fecal microbiota transplantation: donor relation, fresh or frozen, delivery methods, cost-effectiveness. Ann Gastroenterol 2019;32:30-8

12 van Nood E, Vrieze A, Nieuwdorp M, et al. Duodenal infusion of donor feces for recurrent Clostridium difficile. N Engl J Med 2013;368:407-15.

13 Imdad A, Nicholson MR, Tanner-Smith EE, et al. Fecal transplantation for treatment of inflammatory bowel disease. Cochrane Database Syst Rev 2018; 11:Cd012774

14 Allegretti JR, Mullish BH, Kelly C, et al. The evolution of the use of faecal microbiota transplantation and emerging therapeutic indications. Lancet 2019;394:420-31.

15 Kragsnaes MS, Kjeldsen J, Horn HC, et al. Efficacy and safety of faecal microbiota transplantation in patients with psoriatic arthritis: protocol for a 6-month, doubleblind, randomised, placebo-controlled trial. BMJ Open 2018;8:e019231.

16 Coates LC, Conaghan PG, Emery P, et al. Sensitivity and specificity of the classification of psoriatic arthritis criteria in early psoriatic arthritis. Arthritis Rheum 2012:64:3150-5.

17 Kragsnaes MS, Nilsson AC, Kjeldsen J, et al. How do I establish a stool bank for fecal microbiota transplantation within the blood- and tissue transplant service? Transfusion 2020;60:1135-41

18 Jørgensen SMD, Hansen MM, Erikstrup C, et al. Faecal microbiota transplantation: establishment of a clinical application framework. Eur J Gastroenterol Hepatol 2017:29:e36-45.

19 U.S. Department of Health and Human Services. Common terminology criteria for adverse events (CTCAE) version 5.0, 2017. Available: https://ctep.cancer.gov/ protocolDevelopment/electronic_applications/docs/CTCAE_v5_Quick_Reference_5x7. pdf [Accessed 03 Aug 2020].

20 Gossec L, Baraliakos X, Kerschbaumer A, et al. EULAR recommendations for the management of psoriatic arthritis with pharmacological therapies: 2019 update. Ann Rheum Dis 2020:79:700-12.

21 Leung YY, Orbai Ana-Maria, Wit M, et al. Comparing the Patient-Reported physical function outcome measures in a Real-Life international cohort of patients with psoriatic arthritis. Arthritis Care Res 2021;73:593-602.

22 Felson DT, Anderson JJ, Boers M, et al. American College of rheumatology. Preliminary definition of improvement in rheumatoid arthritis. Arthritis Rheum 1995;38:727-35.

23 Maksymowych WP, Mallon C, Morrow S, et al. Development and validation of the spondyloarthritis research Consortium of Canada (SPARCC) Enthesitis index. Ann Rheum Dis 2009;68:948-53.

24 Orbai $\mathrm{A}-\mathrm{M}$, de Wit $\mathrm{M}$, Mease $\mathrm{P}$, et al. International patient and physician consensus on a psoriatic arthritis core outcome set for clinical trials. Ann Rheum Dis 2017;76:673-80

25 Harris PA, Taylor R, Thielke R, et al. Research electronic data capture (REDCap)--a metadata-driven methodology and workflow process for providing translational research informatics support. J Biomed Inform 2009;42:377-81.

26 Mease PJ, Woolley JM, Bitman B, et al. Minimally important difference of health assessment questionnaire in psoriatic arthritis: relating thresholds of improvement in functional ability to patient-rated importance and satisfaction. J Rheumatol 2011:38:2461-5.

27 Selvanderan SP, Goldblatt F, Nguyen NQ, et al. Faecal microbiota transplantation for Clostridium difficile infection resulting in a decrease in psoriatic arthritis disease activity. Clin Exp Rheumatol 2019;37:514-5

28 Lai CY, Sung J, Cheng F, et al. Systematic review with meta-analysis: review of donor features, procedures and outcomes in 168 clinical studies of faecal microbiota transplantation. Aliment Pharmacol Ther 2019:49:354-63.

29 Ruff WE, Greiling TM, Kriegel MA. Host-microbiota interactions in immune-mediated diseases. Nat Rev Microbiol 2020;18:521-38.

30 Chriswell ME, Kuhn KA. Microbiota-mediated mucosal inflammation in arthritis. Best Pract Res Clin Rheumatol 2019:33:101492.

31 Psaty BM, Rennie D. Stopping medical research to save money: a broken PACT with researchers and patients. JAMA 2003;289:2128-31.

32 Colloca L, Barsky AJ. Placebo and nocebo effects. N Engl J Med Overseas Ed 2020;382:554-61. 
33 Kragsnaes MS, Sødergren ST, Kjeldsen J, et al. Experiences and perceptions of patients with psoriatic arthritis participating in a trial of faecal microbiota transplantation: a nested qualitative study. BMJ Open 2021;11:e039471.

34 Rodrigues FB, Ferreira JJ. Strategies to minimize placebo effects in research investigations. Int Rev Neurobiol 2020;153:49-70.

35 Blaser MJ. Fecal microbiota transplantation for dysbiosis - predictable risks. N Eng/ J Med 2019;381:2064-6.

36 Costello SP, Hughes PA, Waters O, et al. Effect of fecal microbiota transplantation on 8-Week remission in patients with ulcerative colitis: a randomized clinical trial. JAMA 2019;321:156-64

37 Paramsothy S, Kamm MA, Kaakoush NO, et al. Multidonor intensive faecal microbiota transplantation for active ulcerative colitis: a randomised placebo-controlled trial. Lancet 2017;389:1218-28
38 Moayyedi P, Surette MG, Kim PT, et al. Fecal microbiota transplantation induces remission in patients with active ulcerative colitis in a randomized controlled trial. Gastroenterology 2015;149:102-9.

39 Paramsothy S, Nielsen S, Kamm MA, et al. Specific bacteria and metabolites associated with response to fecal microbiota transplantation in patients with ulcerative colitis. Gastroenterology 2019;156:1440-54.

40 Cammarota G, laniro G, Kelly CR, et al. International consensus conference on stool banking for faecal microbiota transplantation in clinical practice. Gut 2019;68:2111-21.

41 Zhao HL, Chen SZ, Xu HM, et al. Efficacy and safety of fecal microbiota transplantation for treating patients with ulcerative colitis: a systematic review and meta-analysis. J Dig Dis 2020;21:534-48. 